\title{
Investigating Web Search Strategies and Forum Use to Support Diet and Weight Loss
}

m.c. schraefe

ECS, University of Southampton

UK, SO17 1BJ

mc@ecs.soton.ac.uk

Ryen W. White

Microsoft Research

Redmond, WA 98052, USA

ryenw@microsoft.com

Paul André

ECS, University of Southampton

UK, SO17 1BJ

pa2@ecs.soton.ac.uk

Desney Tan

Microsoft Research

Redmond, WA 98052, USA

desney@microsoft.com

Copyright is held by the author/owner(s).

CHI 2009, April 4 - 9, 2009, Boston, MA, USA

ACM 978-1-60558-246-7/09/04.

\begin{abstract}
Healthcare is shifting from being reactive to preventive, with a focus on maintaining general wellness through positive decisions on diet, exercise, and lifestyle. In this paper, we investigate search behavior as people navigate the Web and find support for dietary and weight loss plans. Inspecting the Web search logs of nearly 2,000 users, we show that people progressively narrow their searches to support their progress through these plans. Interestingly, people that visit online health forums seem to progress through the plans' phases more quickly. Based on these results, we conducted a survey to further explore the roles and importance of online forums in supporting dieting and weight loss.
\end{abstract}

\section{Keywords}

Obesity, diet, weight loss, forums, web search

\section{ACM Classification Keywords}

H5.m. Information interfaces and presentation:

Miscellaneous. J.3. Life and Medical Sciences: Health.

\section{Introduction}

Obesity and excessive weight gain are often attributed with causing a number of related medical conditions and, as of 2002, costing those afflicted and government around 92 billion dollars annually in the U.S. alone [2]. Research has shown that the main method to facilitate 
weight loss is diet [4] and that the use of support mechanisms is key to ongoing diet success [3].

The Transtheoretical Model of Change (TTM) [5], which has been shown to generalize to diet [9], identifies five stages in adopting (or terminating) a habit: Precontemplation, Contemplation, Preparation, Action, and Maintenance. Once in the Action phase, community interaction has been shown to be a key mechanism in maintaining action and offsetting relapse. A recent two-year study on obesity and weight loss showed that participants who remained connected with the support team had the highest success rates [3].

Work in human-computer interaction (HCI) around weight loss has focused on how people use health information sites [8] or benefit from specific health status monitoring tools [1]. In this paper, we investigate how users, trying to formulate good dietary and weight loss plans, search the Web for related information. We investigate the use of online support forums in the process of finding, adopting, and maintaining diets. We also use the TTM to situate these users in their weight loss process and consider strategies to support them.

\section{Examining Web Search Strategies}

At the outset of our research, we studied diet-related browsing trails in Web interaction logs to determine how users forage for diet or weight loss information.

We obtained anonymized querying and browsing behavior logs through a widely distributed Web browser toolbar. The results described in this paper are based on a sample of URL visits during a four-month period from March 2008 to June 2008 for more than 500,000 unique users. From the initial set of logs we extracted over 500 million search trails, each was a temporallyordered sequence of URLs that began with a Web search engine query and terminated with a period of user inactivity of at least $\mathbf{3 0}$ minutes. Studying web search logs let us study users' natural search and browsing behaviors at scale. Contextual inquiry with a focus on failure analysis is an important next step.

We selected trails that began with a query containing at least one relevant keyword (e.g., diet, body mass index, nutrition, etc.) from an author-constructed lexicon, or diet name from www.dietriot.com/dietlist.asp. The presence of such terminology identified users with nutritional interests. To improve coverage, we also assigned Open Directory Project (ODP) labels to trail URLs by starting with URLs that were in the ODP topical hierarchy and backing-off to cover other URLs as necessary [6]. We considered search trails with pages in the 'Health/Nutrition' and 'Health/Weight_Loss' categories as relevant, regardless of their initial query.

Using the query- and URL-labeling procedure we selected around 50,000 users with both nutritionrelated interests. We obtained six consecutive weeks of logs for 1,985 diet/weight-loss users ${ }^{1}$, comprising around 250,000 nutrition-related search trails. For each user, the six-week period began with the first time they issued a nutrition-related query or visited a nutrition-related URL.

To help answer our research question about the effects of online forum use, we divided users into forum and non-forum users. We did this based on whether they visited an online nutrition forum in the six-week period. We conjectured that forum users may seek different support than non-forum users. Results show differences in the search strategies that forum users and non-users employ.

\footnotetext{
${ }^{1}$ Logging errors prevented us from using longer durations without dropping a significant number of users.
} 


\section{Search Log Findings}

\section{Online Behavior}

We analyzed the number of nutrition and diet sites visited, forum use, and the extent to which users stuck with forums. A stabilization in behavior might suggest a progression through TTM phases as diet plans mature.

Over the six weeks, online nutrition forum use increases. That is, few users utilize forums in the first week, but this number grows as time passes. The percentage of users that visit forums is illustrated by the combination of the red and orange bars in Figure 1. This ranges from $2 \%$ of all users in the first week to $22 \%$ of all users in the sixth week. Users become more likely to engage with a forum as time passes.

Figure 1 illustrates the percentage of subjects that use a forum (full red/orange stacked bar) and then revisit the same forum the following week (red portion of stacked bar). Over $50 \%$ of users that visit a forum revisit that same forum the next week. ${ }^{2}$ This shows that once online forum use begins it is highly engaging.

We also studied the number of unique nutrition/diet URLs visited by users per week, and found that it drops consistently over the six weeks (see line graph in Figure 1). This suggests that users are narrowing their diet selections. While the trends are similar for forum users from non-forum users, we see a significant difference from week two onwards in how rapidly each group focuses on a single or a small number of nutrition sites. ${ }^{3}$ Forum users converge on a smaller set of relevant sites more rapidly.

${ }^{2}$ For week six, we calculate this percentage based only on the 1,784 users that reappeared in our logs the seventh week, making this a potentially conservative estimate for week six

${ }^{3}$ Independent measures t-tests, all $t(1983) \geq 2.33$, all $p \leq .02$

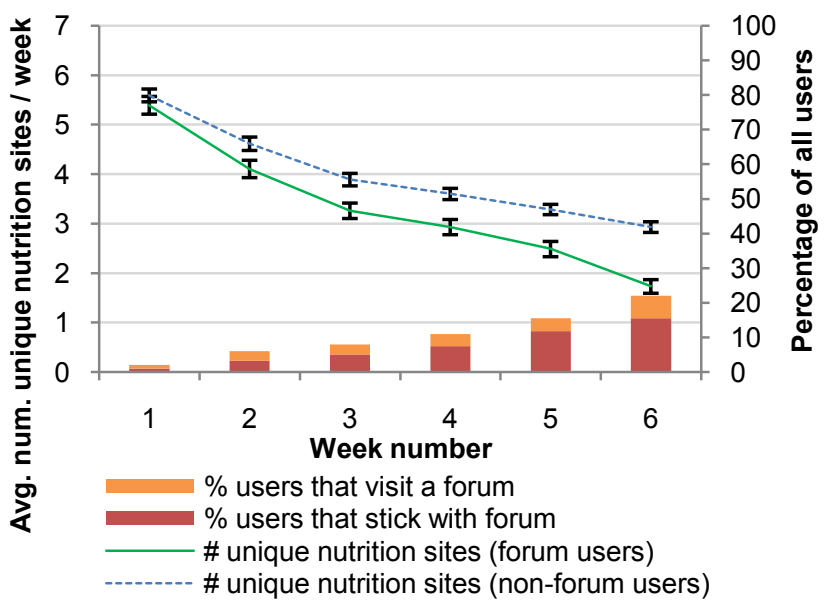

Figure 1. Unique nutrition sites visited and percentage of users visiting and revisiting online nutrition forums.

Search Patterns

To determine whether there were search patterns indicative of progression through TTM phases, we studied the average number of unique diet names (e.g., Atkins, Slim Fast) present in queries and the number of monitoring tool queries (e.g., calorie counting, body fat monitors) issued per week. The findings of this analysis are shown in Figure 2.

The number of unique diets in queries falls over time, suggesting that people settle on particular diets. This is supported by post six-week analysis suggesting that a single - perhaps followed - diet is eventually targeted by most users. Forum users seem to focus on a diet more quickly than non-forum users, with the difference becoming statistically significant in week six. ${ }^{4}$

\footnotetext{
${ }^{4}$ Independent measures t-test, $t(1983)=2.58, p=.01$
} 


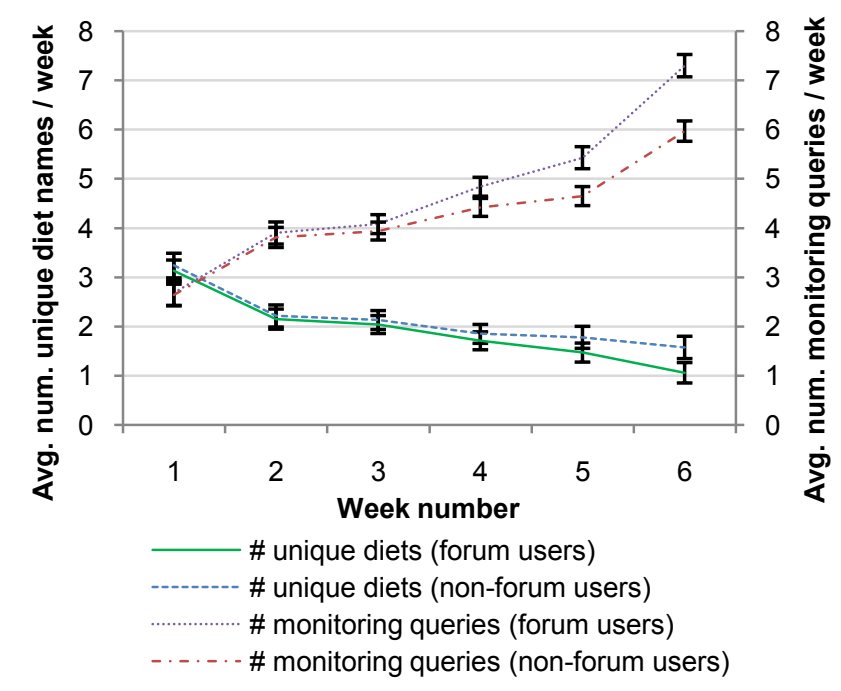

Figure 2. Unique diet names searched for and monitoring queries issued.

The analysis also reveals that the use of health monitoring phrases increases over time, suggesting that users are settling into behaviors that they need to monitor. The differences between forum users and nonforum users are statistically significant from week four onwards. ${ }^{5}$ This suggests that forum users investigate progress monitoring approaches more actively.

Trends in our findings seem to represent a progression through the basic phases outlined earlier. They show that people start with a wide set of mixed queries and progress to focus them, shifting from high-level dietary information needs to more pragmatic implementation details such as monitoring. This is expected, regardless of whether or not people use online forums. The difference between groups, as illustrated by Figures 1

\footnotetext{
${ }^{5}$ Independent measures t-tests, all $t(1983) \geq 2.35$, all $p \leq .02$
}

and 2 , is the efficacy with which this happens. Forum users seem to transition between phases faster.

\section{Specific Query Behavior}

To further explore differences between the forum users and non-forum users' search behavior, we extracted their top-100 popular nutrition-related queries. We removed frequent queries that were common to both lists (e.g., weight watchers, bmi), leaving us with a set of 56 unique nutrition-related queries from each group.

The first noticeable difference between the groups was in query length: forum user queries were on average 3.6 words in length, whereas non-forum users' queries contained on average 1.9 words. This suggests greater focus in the search strategies of forum users. They may know more specifically what they wish to investigate or that they are more motivated to act (i.e., most of their top-20 queries contain the keywords "diet" and "weight loss"). It also suggests that they seek different kinds of information, perhaps because they are engaged in a different task, are at a different TTM phase, or are pursuing a different diet strategy.

Another noticeable between-group difference lay in query semantics. Among forum users diet-related queries comprised the highest percentage $(20 \%)$ is compared with $7 \%$ in the non-forum group. In nonforum users, the highest percentage of queries (18\%) was about supplements or drugs reputed to help burn fat (e.g., single-term queries such as dhea, meridia, Icarnitine, royal jelly, lipodrene, orlistat, chitosan) and a query on bariatric surgery to curb food intake.

The observed differences in query focus may suggest different attitudes toward achieving body composition changes. Diets require a person follow a particular plan that likely involves change of habits. Fat loss supplements or pills are usually seen as approaches in 
lieu of habit change. This potential attitude difference will have an impact on search and potential success strategies, and warrants further study.

\section{Survey to Understand Forum Use}

Our search log analysis suggests that forum users are able to focus on information meaningful to their goals sooner than non-forum users. To understand forum use, we deployed a survey to eight different online health forums, all with greater than 3,000 active users.

Over a one week period, 44 people ( 22 male, 18 female, 4 unknown) between 18 and 69 years old (median in 35-45 range) responded to the survey. After calculating their body-mass index (BMI), 43\% of respondents indicated they were of normal weight, $32 \%$ were overweight, and $20 \%$ were obese. When asked about their goals, respondents of average weight wanted to slim down, eat more healthily, determine whether websites made weight loss complicated, and improve their strength/weight ratio. Overweight and obese respondents were usually seeking to lose weight.

Forum dieters seemed successful, with $97 \%$ stating that they were satisfied with their progress.

Interestingly, respondents reported attempting around two diets on average, settling on the second one. $50 \%$ reported finding their diet through a search engine or health-related portal such as MSN Health, and 35\% of people were helped to their current diet through an online forum. Interestingly, $66 \%$ reported knowing someone on the same diet and $42 \%$ report finding the diets through direct recommendations. Most dieters (75\%) reported looking at multiple sources before starting the diet. Authoritative sources were important and over $70 \%$ searched for the diet's own website to learn more about it.
Once they picked a diet, respondents reported continued use of online tools to get more information or to track progress. $58 \%$ said that forums were their most frequent source of diet information, with $80 \%$ checking online support networks daily or more frequently. Respondents with a normal BMI reported visiting forums daily or weekly; overweight or obese respondents tended to visit the forum greater than once a day. Forums provide an important resource, especially for those with severe weight problems.

Revisitation is also an important part of respondents' web search behavior (as suggested by Figure 1). When searching for diet information, $49 \%$ of respondents primarily searched previously-visited websites for new content. In contrast, the others searched mainly to cross-check, confirm, or find new information.

We also found monitoring progress to be an important aspect of diet-related behavior. To track their progress, $53 \%$ of respondents keep a daily diary of what they eat, $78 \%$ of who store them electronically. $52 \%$ of the respondents weigh themselves once per day, and $84 \%$ of respondents weigh themselves at least once per week. $64 \%$ of respondents use online calorie tracking tools and $61 \%$ of respondents would use a weightrecording scale, if one existed.

Interestingly, successful dieters also implicitly treated dieting as long term activity, and tended to stick with the same diet over the long-term. $72 \%$ of respondents had been on their diet for over three months, and $50 \%$ for over a year. $63 \%$ of respondents report looking back at diet records of over a year of more.

\section{Discussion and Conclusion}

The Web search logs reveal interesting trends in the progression of search queries as users mature their diet and weight loss investigations. For example, some 
users seem to become more likely to use a forum as time passes, with forum users reporting a high sense of progress in their dietary practice. Results also indicate a progressive narrowing of search for different regimens, and an increased interest in progress monitoring tools. In fact, survey results show the importance of these tools within the forum and related online resources such as diet and calorie counter sites and diet logging. But this is not easy. At least one survey respondent stated that the effort in dieting should lie in the diet itself not the tracking and that current tools were too difficult to find and use.

Results suggest that users who visit forums seem to progress through the phases more quickly than their counterparts who do not. On average they start the trend toward focusing on a single diet sooner, and also seem to explore different factors, which can loosely be tied to the success of their regiments. For example, they seem to search less for weight loss pills than nonforum users. However, this could also be attributed to the forum users having different outlets than search from which to obtain their information; they may be tapping forum resources instead. Drawing clearer links between online activity and actual behavior remains future work.

While these results are preliminary, the trends seem sufficiently compelling to warrant further study. We are particularly interested in how keyword search might be augmented with associated information. For instance, we have seen that forum use seems to improve speed of diet focus. Likewise, forum users also reported frequenting different types of website: official diet websites, personal sites about a diet, forums, etc. How this knowledge of successful information patterns might be integrated into search is no small challenge: how could we convey the different types of website available (including related resources such as forums) and what others have found them useful for, and would this associated information accelerate knowledge discovery? We hypothesize that addressing these challenges for revitalized search generalizes beyond diet support. Our goal is to develop techniques to help users discover and integrate various online resources that would help users attain information and skills to move through the phases of successful change more effectively.

\section{References}

[1] Consolvo, S., et al. (2008). Flowers or a robot army? Encouraging awareness and activity with personal, mobile displays. Proc. UBICOMP, in press.

2] Finkelstein, E.A., Fiebelkorn, I.C. \& Wang, G. (2003). National medical spending attributable to overweight and obesity: How much, and who's paying? Health Affairs. [3] Jakicic, J.M., Marcus, B.H. \& Lang W. (2008). 24Month effects of exercise dose and intensity on weight loss in overweight, sedentary women: a randomized trial. Arch. Intern. Med, 168: 1550-1559.

[4] Lockwood, C.M., et al. (2008). Minimal nutrition intervention with high-protein/low-carbohydrate and lowfat, nutrient-dense food supplement improves body composition and exercise benefits in overweight adults: A randomized controlled trial. Nut. and Metab., 5:11

[5] Prochaska, J.O. \& Velicer, W.F. (1997). Behavior change: The transtheoretical model of health behavior change. Amer. J. of Health Promotion, 12(1): 38-48.

[6] Shen, X., Dumais, S. \& Horvitz, E. (2005). Analysis of topic dynamics in web search. Proc. WWW, 1102-1103. [7] Sillence, E., Briggs, P., Fishwick, L. \& Harris, P. (2004). Trust and mistrust of online health sites. Proc. SIGCHI, 663-670.

[8] Toscos, T., Faber, A., An, S. \& Gandhi, M.P. (2006). Chick clique: persuasive technology to motivate teenage girls to exercise. Proc. SIGCHI, 1873-1878.

[9] Wright, J.A., et al. (2008). Testing the predictive power of the transtheoretical model of behavior change applied to dietary fat intake. Health Education Research. 